\title{
Context Modeling with Bayesian Network Ensemble for Recognizing Objects in Uncertain Environments
}

\author{
Seung-Bin Im, Youn-Suk Song, and Sung-Bae Cho \\ Department of Computer Science \\ Yonsei University \\ 134 Shinchon-dong, Sudaemoon-ku, Seoul 120-749, Korea \\ \{envymask, corlary\}@sclab.yonsei.ac.kr, sbcho@cs.yonsei.ac.kr
}

\begin{abstract}
It is difficult to understand a scene from visual information in uncertain real world. Since Bayesian network $(\mathrm{BN})$ is known as good in this uncertainty, it has received significant attention in the area of vision-based scene understanding. However, BN-based modeling methods still have the difficulties in modeling complex relationships and combining several modules, as well as the high computational complexity of inference. To overcome them, this paper proposes a method to divide and select the $\mathrm{BN}$ modules for recognizing the objects in uncertain environments. The method utilizes the behavior selection network to select the most appropriate BN modules. Several experiments are performed to verify the usefulness of the proposed method.
\end{abstract}

\section{Introduction}

Scene understanding, recognizing contexts to understand the class of scene or situation, is a very difficult and largely unsolved problem. When running a scene understanding process, visual information may be uncertain due to motion blur, bad lighting condition, etc. We need the recognition methods that are robust in uncertainty.

Since Bayesian network (BN) is good for modeling uncertain domain, it has received significant attention in the area of vision-based scene understanding [1, 2]. However, if we adopt BN techniques for real world scene understanding, there are some problems we must solve. At first, modeling complex relationships of real world is difficult and the nodes that represent relationships require large amount of computation [3]. Moreover, it is difficult to combine various goal-specific BN modules. Therefore, a novel method is required for modeling and combining BNs without any significant increase of computation.

This paper proposes a practical method of objects recognition for service robots. The method utilizes the behavior selection network (BSN) to effectively combine the pre-designed $\mathrm{BN}$ modules and the activity-object context BN modules for the prediction of target objects and the control of robot behaviors to find objects in several places. 


\section{Context Modeling and BN Ensemble Based on Domain Knowledge}

\subsection{BN Design Method Based on Domain Knowledge of Occluded Object}

We propose a method of $\mathrm{BN}$ design for reasoning the occluded objects using the object relationships based on activity. Fig. 1 shows the process of designing them as tree structure based on common-cause principle. Constructed $\mathrm{BN}$ modules are composed of activity, class and primitive nodes. The $\mathrm{BN}$ is a singly-connected structure like tree, and common-cause structures are used for sub-trees: it is a sort of causal relationships among three nodes. It has two nodes that have another node as a parent in common. This structure allows us to represent the relationship of objects more easily and precisely [4]. The process of designing BNs for occluded objects is a simple causal chain because it is possible to assign the parameters to each node for representing the relationship between them.

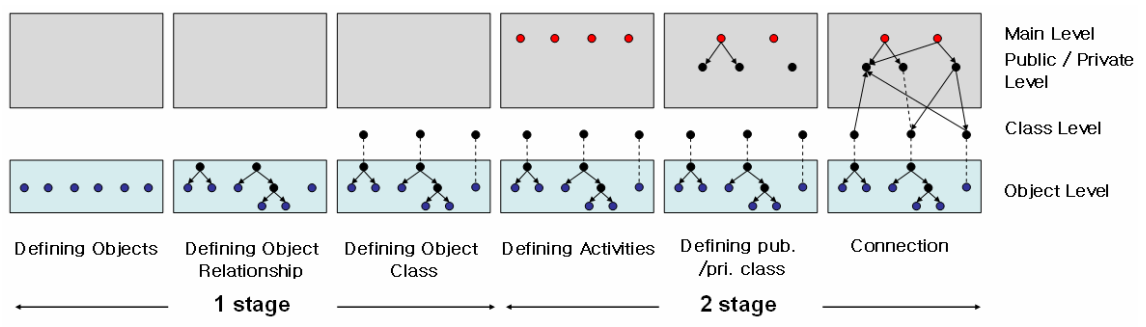

Fig. 1. The process of designing BN modules for occluded objects

\subsection{Behavior Selection Network for BN Ensemble}

Behavior selection network accomplishes the objectives through action selection and state transition. Usual input for BSN is sensory information, but we connect the $\mathrm{BN}$ inference results to sensor input for more accurate environmental perception. We have modified the original definition of BSN inference [5] with the following equations.

At the forward propagation, activation $a_{1}$ is

$$
\begin{aligned}
& \Delta a_{1}=\sum_{i=1}^{n} f\left(a_{s_{i}}\right)+\sum_{k=1}^{m} f\left(a_{B_{k}}\right), \text { where } f\left(a_{B_{k}}\right)=\phi \times a_{B_{k}} \\
& f\left(a_{B_{k}}\right)=P\left(y_{k} \mid \text { Parents }\left(y_{k}\right), \text { Child }\left(y_{k}\right)\right) \text {, where } y_{k} \text { is the query node of each BN }
\end{aligned}
$$

Here, $n$ means the number of sensors, $m$ means the number of inferential results that can be the input of BSN, and $f(a)$ is the Bayesian inference function of each BN. BSN module combines the pre-designed BNs (for recognizing places and objects) and activity $\mathrm{BN}$ that was constructed by the proposed method. 


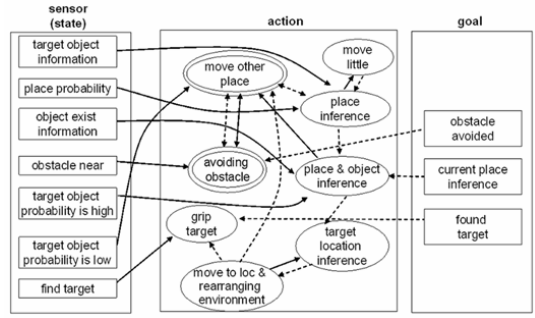

(a)

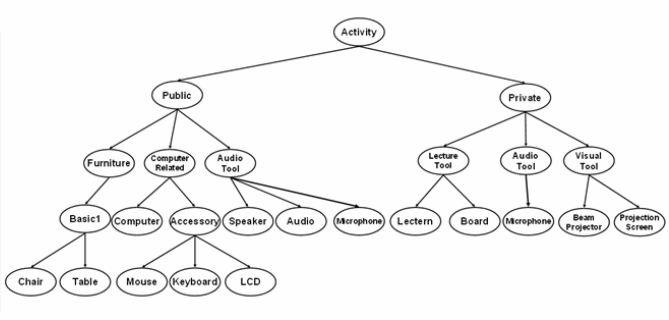

(b)

Fig. 2. (a) Structured behavior selection network, (b) Constructed BN for objects that have the relationship with the activity of 'presentation'

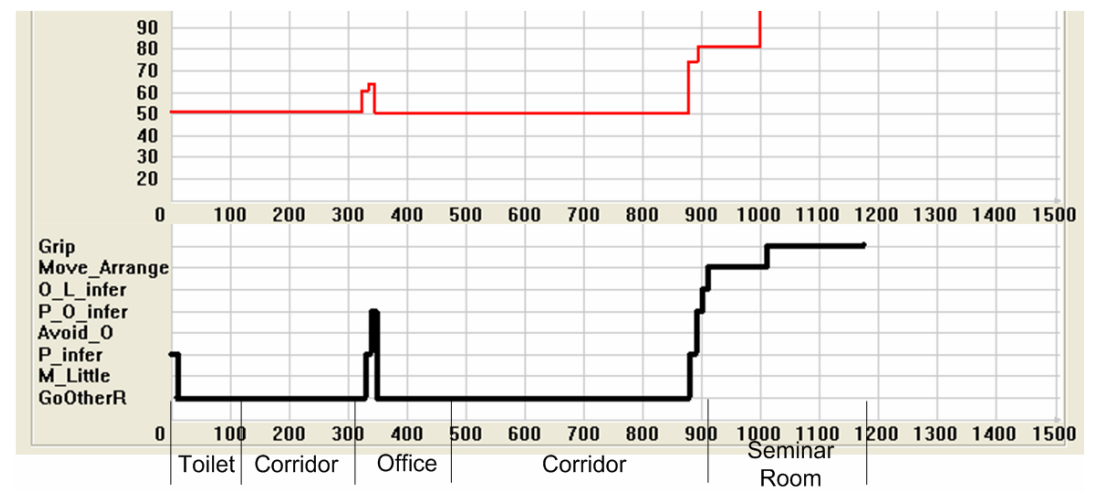

Fig. 3. Experimental results of finding beam-projector, occluded target object. Upper thin line shows the object existence probability and under thick line shows the state transition.

\section{Experimental Results}

The task of the experiments is to let the service robot find occluded objects in indoor environments and design Bayesian networks according to the proposed method. In the experiment, the robot wanders around four places (toilet, corridor, office, seminar room) and finds a beam projector, the target object occluded. In this paper, we have used simulated experimental environment, and randomly generated place probabilities in each places according to real world probability distribution that extracted from vision data. Fig. 3 shows one of the experimental processes that were executed in the webot simulator. Upper graph shows the change of object existence probability and lower graph depicts the states selected by BSN. If the inferred probability is lower than threshold of $70 \%$, deeper-level inference is triggered. We confirmed that BSN module could find target objects precisely and efficiently: The BSN produced $100 \%$ accuracy in ten repeated runs.

Fig. 4 shows the result of the second experiment: prediction of beam-projector existence in contextually similar places. That is shown under threshold of $70 \%$ until the robot finds five objects with the activity-object context $\mathrm{BN}$ in the BSN module. 
Predictions seem reasonable except one case. It can be seen that false-positive error is likely to occur in the similar environment from the result of accumulating evidences.
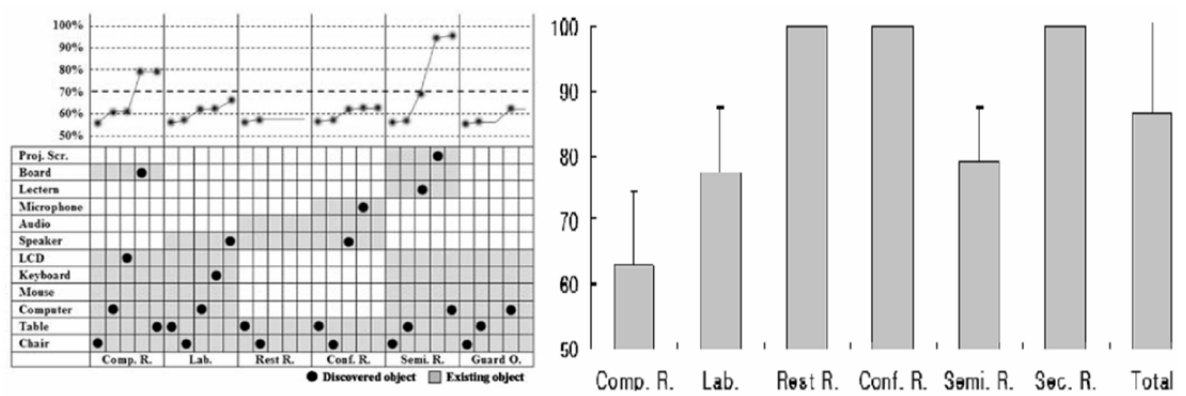

Fig. 4. The result of predicting an occluded-object (beam- projector) in six places (computer room, laboratory, rest room, conference room, seminar room, and security room)

\section{Concluding Remarks}

In this paper, we proposed a context modeling method for scene understanding (especially, object recognition) and intelligent service of robot based on image. We use the BSN for properly combining several types of BN modules used for inferring places and occluded objects as well as controlling robot behavior for its goal. In addition, we proposed a BN modeling method for inferring place and occluded objects. The experiments show the usefulness of the proposed method. For the future works, we will extend the scope of domains and apply the method to the real robots.

Acknowledgments. This work was supported by the Korea Science and Engineering Foundation (KOSEF) through the Biometrics Engineering Research Center (BERC) at Yonsei University.

\section{References}

1. B. Neumann, A Conceptual Framework for High-Level Vision, Bericht, FB Informatik, FBIHH-B245/03, 2003.

2. M. Marengoni, A. Hanson, S. Zilberstein and E. Riseman, "Decision making and uncertainty management in a 3D reconstruction system," IEEE Trans. Pattern Analysis and Machine Intelligence, vol. 25, no. 7, pp. 852-858, 2003.

3. M. Neil, N. Fenton and L. Nielson, "Building large-scale Bayesian networks," The Knowledge Engineering Review, vol. 15, no. 3, pp. 257-284, 2000.

4. Y.-S. Song, S.-B. Cho, and I.-H. Suh, "Activity-object Bayesian networks for detecting occluded objects in uncertain indoor environment," Int. Conf. on Knowledge-Based Intelligent Information \& Engineering Systems, vol. 3683, pp. 937-944, 2005.

5. K.-J. Kim, S.-B. Cho, "BN+BN: Behavior network with Bayesian network for intelligent agent," Lecture Notes in Artificial Intelligence, vol. 2903, pp 979-991, 2003. 